Bridgelall, R., Chia, L., Bhardwaj B., Lu, P., Tolliver, D., and Dhingra, N. (2019). Enhancement of Signals from Connected Vehicles to Detect Roadway and Railway Anomalies. Measurement Science and Technology, November 25, 2019. DOI: 10.1088/1361-6501/ab5b54.

\title{
Enhancement of Signals from Connected Vehicles to Detect Roadway and Railway Anomalies
}

Raj Bridgelall, Ph.D.*

Assistant Professor and Program Director

Department of Transportation, Logistics and Finance, North Dakota State University

NDSU Department 2880 P.O. Box 6050, Fargo, ND 58101-6050

Phone: (408) 607-3214; Email: raj@ bridgelall.com; ORCID: 0000-0003-3743-6652

\section{Leonard A. Chia}

Research Assistant

Department of Transportation, Logistics and Finance, North Dakota State University

NDSU Department 2880 P.O. Box 6050, Fargo, ND 58108-6050

Phone: (701) 630-9377; E-mail: leonard.chia@ @ndsu.edu; ORCID: 0000-0001-9174-4969

\section{Bhavana Bhardwaj}

Research Assistant

Department of Computer Science, North Dakota State University

NDSU Department 2880 P.O. Box 6050 Fargo, ND 58108-6050,

Phone: (701)-729 -1647; E-mail: bhavana.bhardwaj@ndsu.edu; ORCID: 0000-0002-4379-1565

Pan Lu, Ph.D.

Associate Professor and Associate Research Fellow

Department of Transportation, Logistics and Finance, North Dakota State University

NDSU Department 2880 P.O. Box 6050, Fargo, ND 58101-6050

Phone: (720)-238-0080; E-mail: pan.lu@ ndsu.edu; ORCID: 0000-0002-1640-3598

Denver D. Tolliver, Ph.D.

Director

Upper Great Plains Transportation Institute, North Dakota State University

NDSU Department 2880 P.O. Box 6050, Fargo, ND 58108

Phone: (701) 231-7190; Email: denver.tolliver@ ndsu.edu; ORCID: 0000-0002-8522-9394

Neeraj Dhingra

Research Assistant

Department of Transportation, Logistics and Finance, North Dakota State University

NDSU Department 2880 P.O. Box 6050, Fargo, ND 58108-6050

Phone: (701) 729-1641; E-mail: neeraj.dhingra@ ndsu.edu; ORCID: 0000-0001-9970-7185

*Corresponding Author 


\begin{abstract}
Frequent network-wide monitoring of the condition of roadways and railways prevent fatalities, injuries, and financial losses. Even so, agencies cannot afford to inspect vast transportation networks using present methods. Therefore, the idea of using low-cost sensors aboard connected vehicles became appealing. However, low-cost sensors introduce new challenges to improve poor signal quality which causes detection errors. Common approaches apply computationally complex filters to individual signal streams, which limits further improvements. This paper presents a method that combines signals from each traversal in a manner that leads to ever-increasing signal quality. The proposed method addresses the challenges of poor accuracy and precision of position estimates from global positioning system (GPS) receivers, and errors from the non-uniform sampling of low-cost accelerometers. The result is improved signal quality from a $20 \%$ improvement in signal alignment over GPS and a 90-fold enhancement in distance precision.
\end{abstract}

Keywords: Ensemble Averaging; Feature Extraction; GPS Errors; Low-Cost MEMs; Pothole Detection; Railroad Track Geometry; Signal Alignment 


\section{Introduction}

The economic health of a nation relies on the efficient and safe movement of people, goods, and waste across a network of roadways and railways. This network can be vast in some countries. Roadway and railway anomalies that develop over time can be anything on the surface that increases the roughness of a ride. Roadway anomalies such as potholes can puncture tires, bend wheels, damage suspension systems, and cause other failures that result in accumulated expenses exceeding billions of dollars annually (El-Wakeel, et al. 2018). Swerving to avoid anomalies such as potholes can lead to roadway accidents (Jo and Ryu 2015). Railway anomalies such as broken tracks have led to large financial losses from many derailments (Aldrich 2018). Therefore, more frequent and network-wide monitoring to detect and fix anomalies can help reduce the risk of harm and financial losses. However, inspections to find roadway and railway anomalies require the use of specially equipped vehicles and trained inspectors. For example, the cost of current automated highway inspection methods can range from $\$ 28$ to $\$ 115$ per mile, which limits the affordability of more frequent monitoring and greater network coverage (Pierce and Weitzel 2019). Consequently, anomalies such as frost heaves that appear and disappear between monitoring cycles can go unnoticed until they cause significant damage.

An emerging strategy to increase monitoring frequency and coverage is to use sensors available onboard regular vehicles. Such sensors include GPS, accelerometers, speed, and timers. Deviations from ride smoothness produce inertial events that indicate the presence of roadway or railway anomalies. For example, potholes or frost heaves on pavements or broken rails or ties on railroad tracks can produce sudden vertical accelerations. Lateral accelerations or rolling could indicate the presence of irregular railroad track geometry. Sensors aboard regular vehicles can produce inertial signals and geospatial position data that make it possible to detect anomalies at significantly reduced costs. The recent widespread deployment of a sensor-based train safety system (GAO 2019) and the push to integrate standards-based wireless communications into all roadway vehicles (USDOT 2015) present an opportunity to standardize the approach across the entire network of roadways and railways. However, the high cost of sensors could outweigh the benefits. Therefore, the goal of this research is to develop a method that can utilize the data from very lowcost sensors such as the accelerometers and Global Position System (GPS) receivers that are ubiquitous in smartphones. Figure 1 is a graphical overview of this work.

\subsection{Low-Cost Sensor Challenges}

The typical low-cost inertial sensor integrates three axis accelerometers and three axis gyroscopes into a microchip. However, they present several issues in signal detection. A first issue is that they sample at a non-uniform rate (Stiros and Psimoulis 2012). Figure 2 is a histogram of the sample period for an accelerometer embedded in a smartphone - the iPhone $6 \mathrm{~S} \circledast$, set to sample at its maximum rate. As observed, the primary mode was approximately 11 milliseconds, which is equivalent to a sample rate of approximately 90 hertz. However, the sample period varied between 1 and 18 milliseconds. When the sensor samples at those larger intervals, the measurement gaps could miss important inertial events, especially when traveling at higher speeds. Previous work found that the inertial sample rate must be at least 64 hertz for the reliable detection of roadway anomalies (Bridgelall 2014).

A second issue is that sensor noise and suspension system damping can reduce signal quality and sensitivity to inertial events, consequently increasing detection errors (Ma, Xie and Gan 2018). A third issue is that traversing the same anomaly at different speeds will produce different signal 
intensities. For example, the mechanical filtering action of a suspension system can reduce the roughness intensity produced from traversing a narrow bump at a high speed but increase that intensity at a lower speed (Bridgelall, Rahman, et al. 2019). Similarly, the roughness intensity from traversing a wide bump increases with speed.

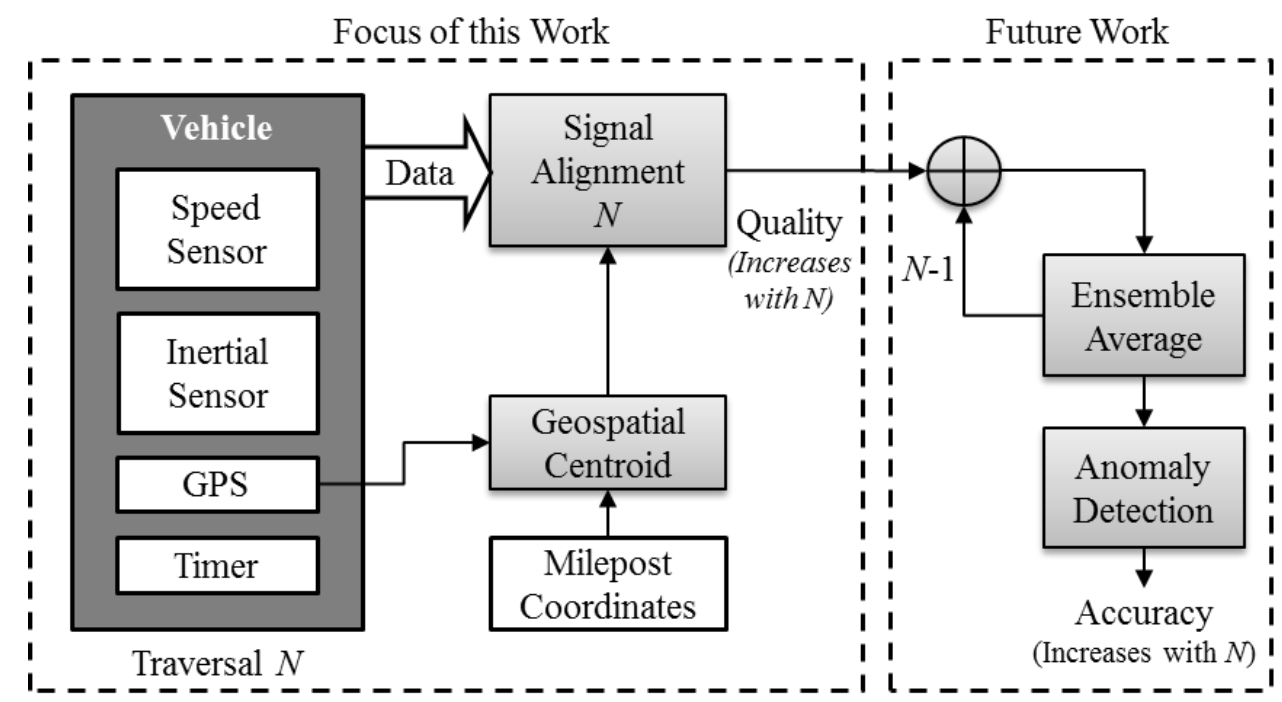

Figure 1. Graphical overview of the development in this paper.

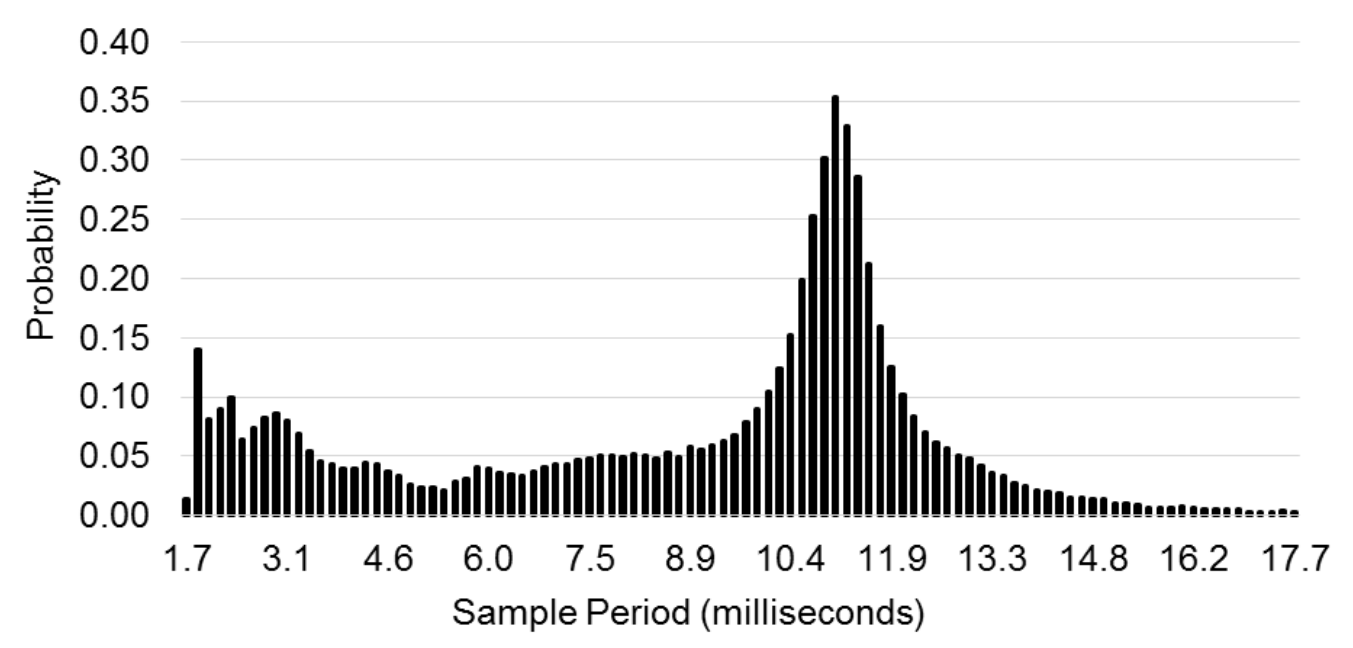

Figure 2. Sample period distribution for the inertial sensor.

\subsection{Proposed Solution}

This paper proposes a solution to improve signal quality and reduce detection errors by combining the inertial signals from multiple traversals of a road or rail segment at any speed. Fundamentally, averaging reduces noise in the composite signal because noise is uncorrelated (C.-T. Chen 2004). Averaging also enhances signals because of their correlation. The proposed strategy is to ensemble average the inertial signals from the same position along the traversal path. Ensemble averaging 
computes the mean value of the inertial signal within a given distance window, for example, every centimeter. Consequently, the ensemble averaging will increase the quality of the composite signal with each additional data stream $N$, as illustrated in Figure 1. Another benefit of the approach is that the signals from traversals at different speeds will increase the probability of detecting an anomaly that may be undetectable at other speeds. Hence, this approach is well-suited for a connected vehicle environment that produces data from many traversals per hour.

Unfortunately, the proposed solution presents new challenges. It requires that the sensor system tag each inertial sample with a precise position so that the algorithm can align the positions of multiple signals before taking the ensemble average. Connected vehicles use the Global Positioning System (GPS) to provide position updates of their travel path, which presents several issues (Bajaj, Ranaweera and Agrawal 2002). The first issue is that standard GPS receivers provide position updates approximately once per second (Hunter, et al. 2009). Given an inertial sensor that samples at 64 hertz on average, the GPS coordinates will update after every group of 64 inertial samples. Therefore, the system will, on average, tag blocks of 64 inertial samples with the same GPS coordinates. This will result in a poor position resolution and consequently large errors in signal alignment. Alternative devices such as inertial measurement units (IMU) can estimate missing GPS coordinates by adding a Kalman Filter to predict samples based on inertial inputs (Gikas and Perakis 2016). However, such devices are not generally available onboard regular vehicles and trains because they are significantly more expensive, and they suffer from ever-increasing drift bias. Therefore, this work focuses on standard low-cost GPS receivers.

The second issue is that position updates among traversals will be spatially asynchronous. That is, the GPS updates from each traversal will be at different points along the path so some traversals will not sample the position of an important inertial event. The third issue is that the standard deviation of the position estimates from GPS receivers is three to five meters along the travel direction (Hughes 2016). A fourth issue is that the surface position error is in two dimensions which results in GPS position updates that are not on the travel path. The fifth issue is that non-lineof-sight (non-LOS) conditions caused by clouds, trees, or tunnels could block the reception of GPS signals in some locations (Groves, Wang and Ziebart 2012). Dynamic multipath effects from satellite signal bounces can also create outliers in the geospatial position estimates (Moschas, Psimoulis and Stiros 2013). Therefore, a data cleaning process is needed to identify and remove data associated with such outliers.

Even if a GPS can provide a higher sample rate, a higher position accuracy, and operate in non-LOS conditions, any errors in their position estimate and asynchronous position updates will result in position misalignment of the inertial signals. Therefore, an ability to improve signal alignment will allow the ensemble averaging to continuously enhance the quality of the composite signal by reducing noise and increasing the precision of the position estimate. Figure 3 helps to demonstrate the signal misalignment issue. The signals shown are the simulated elevation profile from traversing a single isolated bump. The signals (R1 to R3) in Figure 3a are from three identical speed traversals with uniform and spatially synchronous sampling. The function that simulates the elevation profile of a bump is from the class of radial basis functions in mathematics, and is defined as

$$
R(r)=e^{-[\epsilon(r-\rho)]^{2}}+\eta
$$

where $r$ is the distance in meters from a reference position on the traversal path, $\varepsilon$ is the shape parameter that sets the width of the bump, $\rho$ is the distance of the peak, and $\eta$ is noise from random 
vibrations and electrical interference. Figure 3a shows the effect of errors in position tagging the inertial samples of different traversals. Figure $3 b$ shows the effect of ensemble averaging the aligned and non-aligned elevation profiles at each distance point. Signal alignment means that the signal peaks occur at the same distance position. Ensemble averaging reduces noise in both cases because noise is uncorrelated.

Although obvious, it is important to generalize that in addition to the noise reduction of the composite signal, any improvement in signal alignment will improve the accuracy of detecting an anomaly that produced a peak inertial event (PIE). The rate of error reduction as a function of the number of signal streams combined depends on the amount of misalignment, the width of the PIE, and the noise level in each signal. The possibilities are infinite. Nevertheless, regardless of the signal alignment achieved, noise in the composite signal will continue to reduce with each additional signal averaged because of the uncorrelatedness property of noise. Although the signal quality will improve with each additional signal averaged, the rate of improvement will depend on the alignment accuracy achieved by the algorithm. The simulated example illustrates a scenario that demonstrates this benefit.
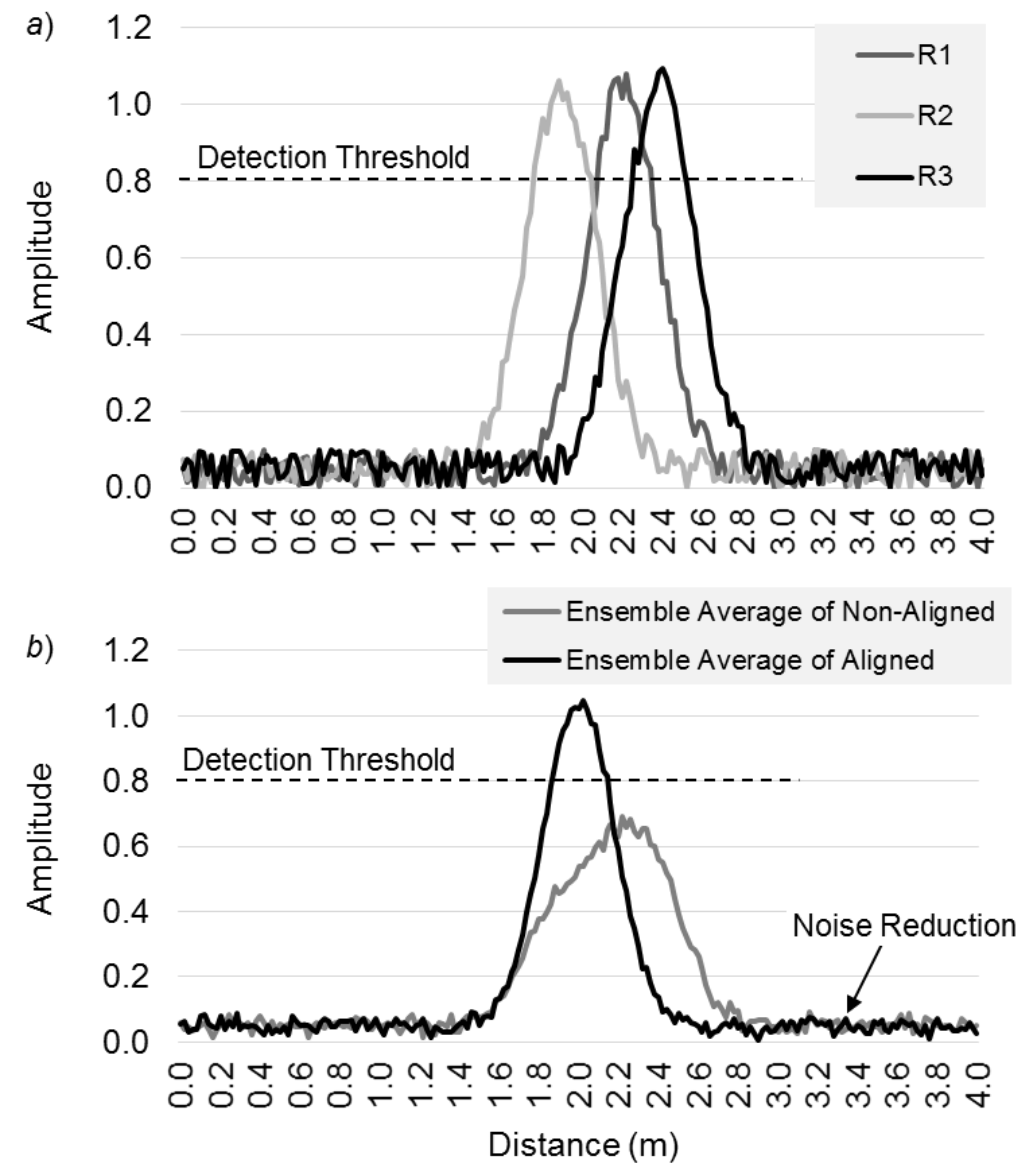

Figure 3. Effect of combining aligned and non-aligned signals.

The simulation shows that for signals with $10 \%$ noise, the ensemble averaging of just three signals reduces the noise in the composite signal by a factor of two. However, a misalignment spread of 0.7 
meters for a PIE signal that is one meter wide decreases the peak of the composite signal by onethird, biases the peak by 0.4 meters, and nearly doubles the width of the PIE. The consequence is a decrease in both the accuracy and precision of estimating the position of an anomaly that produced the PIE. For a scenario of setting the detection threshold for a PIE to 0.8, this amount of misalignment, noise level, and number of signals combined would have resulted in a false negative.

The primary objective of this research is to invent and present an alternative means of distance tagging that both reduces the reliance on GPS position estimates within a traversal and increases position resolution to improve signal alignment. The contribution of this paper is four methods to achieve that objective, and an evaluation and demonstration of their relative performance by using data collected from a sedan.

The organization of the remainder of this paper is as follows: Section 2 discusses related work that investigated the use of sensors aboard connected road and rail vehicles. Section 3 describes the development of the signal alignment algorithms, the data collected to measure misalignment, a reference method to evaluate non-GPS errors, and the statistical methods used for performance evaluation. Section 4 discusses the results in terms of the statistical distributions of a PIE from a known ground truth. Section 5 discusses some surprising observations and offers some practical considerations for using the algorithm. Section 6 provides some concluding remarks about the findings and comments on future work.

\section{Literature Review}

There is general realization that in the future, the Internet of Things (IoT) will lead to a substantial reduction in the cost of connected sensors that can monitor vehicle dynamics (Medeiros, Valente and Nepomuceno 2018). However, few recognize that using low-cost sensors aboard vehicles presents measurement challenges because of uncertainties in speed, weight, and loading conditions (Dertimanis, et al. 2019). The future of IoT will include connected vehicles of all types. The concept of using sensors on connected vehicles to monitor roadway condition gained popularity only within the past five years (Bridgelall 2014) (Dennis, et al. 2014). However, there is a gap in the literature about the use of low-cost sensors on trains to monitor the condition of railways (Bernal, Spiryagin and Cole 2018).

Related research explored the potential benefits of using coupled GPS and inertial sensors with specialized trolleys to detect track geometry issues (Chen, et al. 2018). One group demonstrated good results of estimating track longitudinal profile by processing signals from accelerometers attached to a train bogie (OBrien, et al. 2018). A Portugal study found that the standard deviations of the longitudinal track deviations correlated with the measured vertical accelerations at a level of 0.85 (Paixão, Fortunato and Calçada 2019). A recent literature review found that all past research focused on correlating the inertial signals with ground truth measurements or on techniques to improve estimations from single traversals (Chia, et al. 2018). None of the past work combined the inertial signal streams from multiple traversals in a connected vehicle environment to enhance signal quality.

\section{Methods}

The strategy developed to position align the inertial signals is to replace the GPS position tags with higher resolution distance tags and to identify the starting position along the traversal path. Enhanced distance resolution is achievable by interpolation using the instantaneous speed and sample periods obtained from the onboard sensors. The inertial sample at the starting position $d_{0}$ of a traversal is set to distance zero, and subsequent samples are located at the accumulated distance 


$$
d_{n}=d_{n-1}+v_{n} \times \Delta \tau_{n}
$$

where $n$ is the sample number, $v_{n}$ is the instantaneous speed logged for that sample instant, and $\Delta \tau_{n}$ is the sample period at that sample instant. Interpolating distance with a sample rate that is 90 times the update rate of GPS effectively achieves a precision enhancement of distance along the traversal path that is as many times greater. The high-resolution differential time interpolation produces in a highresolution distance signal that allows the ensemble averaging to compute signal means within small but fixed distance windows along the traversal path, for any vehicle, traveling at any speed, and sampling at any rate. After interpolation, each alignment method needed to identify the first and last inertial sample of each traversal in a manner that produces maximally aligned signals. This section develops and evaluates four methods, using data collected from a sedan.

\subsection{Data Segmentation}

Table 1 shows the format of the data collected using an iPhone $6 \mathrm{~S} \circledast$ mounted onto the dashboard of a sedan. The dataset contains inertial signals from 53 traversals of a road segment that contained a single isolated bump. Driving over the bump produced a consistently large PIE. The columns from left to right are time in milliseconds, vertical acceleration in $\mathrm{g}$-force, instantaneous traversal speed in meters-per-second, latitude in degrees, and longitude in degrees. As observed in Figure 2, the primary mode of accelerometer sampling was approximately 90 hertz and the variance from it was relatively large. Since the GPS updated at approximately 1 hertz, the latitude and longitude remained unchanged for blocks of inertial samples called GPS blocks.

Table 1. Format of Sensor Data

\begin{tabular}{ccccc} 
Time & Gz & Speed & Lat & Lon \\
\hline 44.142 & -1.057 & 9.586 & 45.263 & -93.711 \\
46.768 & -1.216 & 9.586 & 45.263 & -93.711 \\
50.260 & -1.087 & 9.586 & 45.263 & -93.711 \\
62.927 & -0.854 & 9.586 & 45.263 & -93.711 \\
73.909 & -0.912 & 9.586 & 45.263 & -93.711 \\
86.754 & -0.942 & 9.586 & 45.263 & -93.711 \\
95.669 & -1.001 & 9.586 & 45.263 & -93.711 \\
110.365 & -1.022 & 9.586 & 45.263 & -93.711 \\
118.253 & -1.096 & 9.586 & 45.263 & -93.711 \\
128.695 & -1.013 & 9.586 & 45.263 & -93.711 \\
\hline
\end{tabular}

Figure 4 illustrates the relative position variation of GPS blocks among traversals. The geospatial pre-processing algorithm constructed starting and ending geofences to specify the road or rail segment of interest. The algorithm then extracted the data for all traversals contained within the geofence boundaries. The alignment algorithms subsequently operated on each of the extracted datasets.

The distance interpolation needed at least one GPS block from which to begin. Therefore, all traversals must have at least one GPS block to the left and right of a reference distance. The algorithm achieved this by creating two inner geofences, R0 and R1, to serve as common distance references. Both geofences are perpendicular to the traversal path. The algorithm found the optimum 
position for each geofence by placing $\mathrm{R} 0$ and $\mathrm{R} 1$ at the mid-distance point of the segment, and then moving them outwards in opposite directions according to the optimization:

Maximize $\quad \Delta \mathrm{R}=|\mathrm{R} 1-\mathrm{R} 0|$

subject to

$$
\forall j \in \mathbb{T},\left\{\forall\left\|G_{i j}-\overleftrightarrow{R 0}\right\|^{\perp} \leq 0, \sum_{i=1}^{n}\left[G_{i j}\right] \geq 1\right\}, i=\{1,2, \cdots\}
$$

and

$$
\forall j \in \mathbb{T},\left\{\forall\left\|G_{i j}-\overleftrightarrow{R 1}\right\|^{\perp} \geq 0, \sum_{i=1}^{n}\left[G_{i j}\right] \geq 1,\right\}, i=\{1,2, \cdots\}
$$

This expression states that the set of traversals $\mathbb{T}$ have indices $j$. From the starting geofence of a segment, the $i^{\text {th }}$ GPS block in traversal $j$ is $G_{i j}$. The counter operator $\left[G_{i j}\right]$ returns unity for each GPS block, and the operator $\|\cdot\|^{\perp}$ returns the perpendicular distance from the geodesic position of $G_{i j}$ to the geofence indicated such that distances to the right of the starting reference line are positive. Hence, the distance $\left\|G_{i j}-\overleftrightarrow{R 0}\right\|^{\perp}$ is negative for all samples to the left of R0, which is the first constraint, and the distance is positive for all samples to the right of $\mathrm{R} 1$, which is the second constraint.

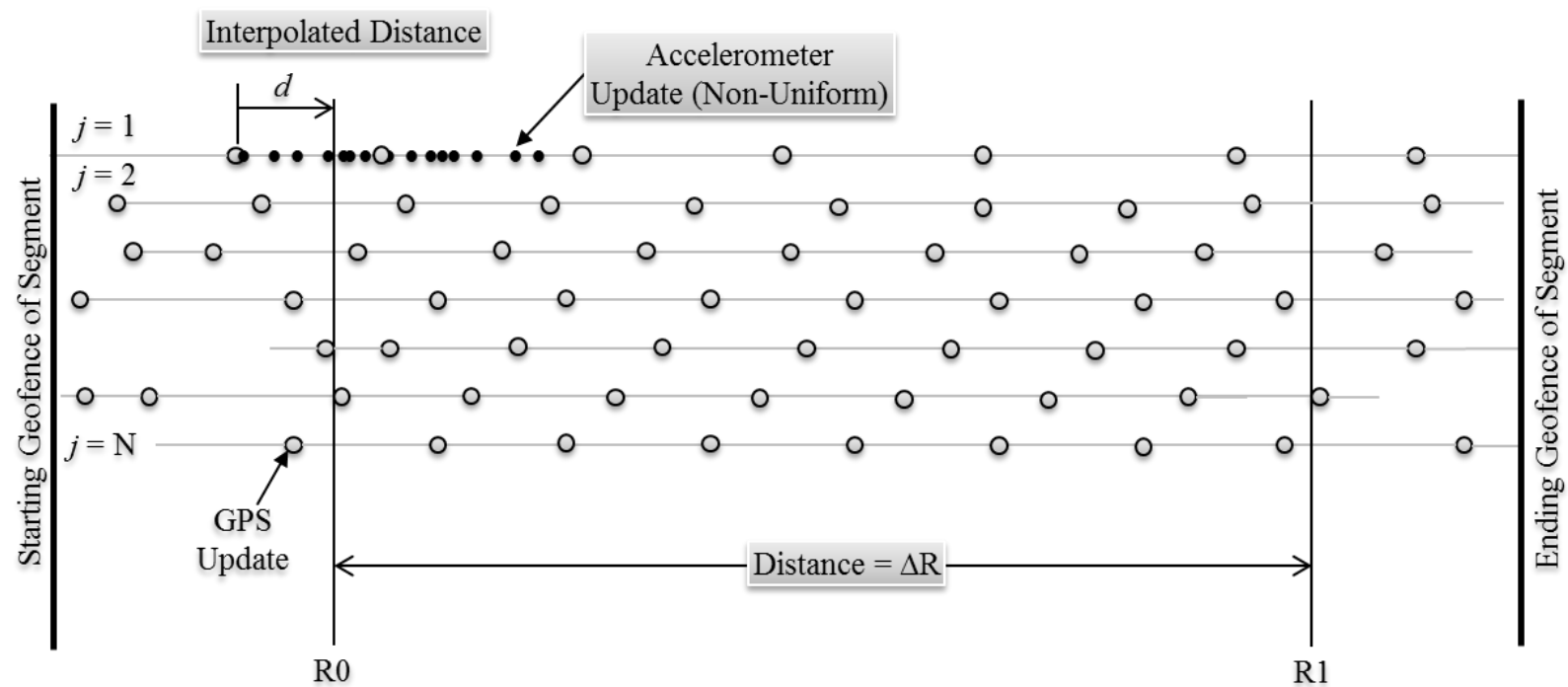

Figure 4. Spatial optimization to establish the segment geofence.

Figure 5a shows how the geospatial coordinate updates for the GPS blocks of the data collected vary between two traversals. The variations are in two dimensions and it is evident that some of the coordinates exit the traversal path. Figure $5 \mathrm{~b}$ is a plot of a small section containing the PIE signal samples from the two traversals shown in Figure 5a. The artificial offset of the second signal by 1.0 g-force is for clarity. The negative peak and the positive peak of the PIE reflects the maxima and minima of the vertical acceleration profile from traversing the bump. The reference distance $\mathrm{d}_{0}=0$ is the first sample of the GPS block that is closest to the left of R0. For this dataset, the GPS updated 
each second consistently. That is, there were no missing GPS updates in this dataset. Hence, the variations in the relative distances of the PIE in this dataset characterizes the misalignment observed in that environment. Figure 5a shows the geospatial positions logged for the PIE of all traversals. The spread was 30.4 meters along the traversal path and 14.5 meters perpendicular to the traversal path. If six standard deviations contained the PIE spread along the traversal path, then the standard deviation of the GPS distance estimate for PIEs was 5.1 meters. A significant benefit of using distances from a common geofence that is perpendicular to the travel path is that it eliminates one dimension of the geospatial errors that estimate positions outside of the travel path.

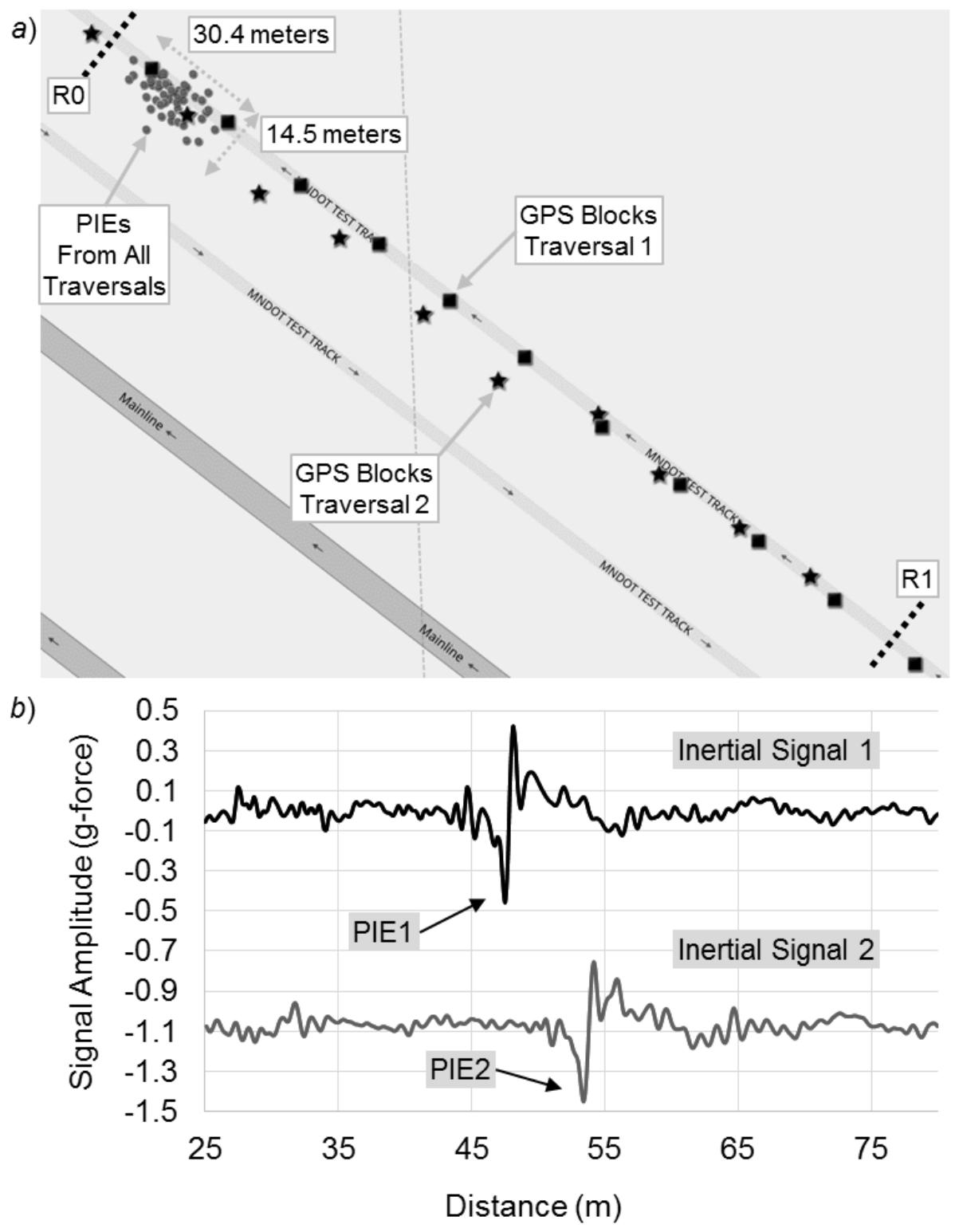

Figure 5. Position variation of the PIE. 


\subsection{Signal Alignment}

The two geofences inspired four tactics to determine the first and last samples of the aligned signals. The names assigned to each algorithm are: Dual Geofence, Midway Midpoint, Centroid Asymmetric, and Centroid Symmetric. The names reflect the core tactic used to identify the first sample for interpolation initialization.

\subsubsection{Dual Geofence}

For all traversals, this method begins by finding the GPS block $G_{\mathrm{L}}$ that is closest to the left of R0 and setting the index of the first sample within the block as the interpolation initialization sample where $n=0$. The algorithm then increments index $n$ from left to right until it identifies the first sample of the aligned signal, which is the index that satisfies the optimization:

Minimize $\quad x_{n}-\left\|G_{\mathrm{L}}-\overleftrightarrow{R 0}\right\|^{\perp}$

subject to

$$
x_{n}=x_{n-1}+v_{n} \times \Delta \tau_{n}, n=\{1,2, \cdots\}
$$

and

$$
x_{0}=0
$$

Next, the algorithm finds the GPS block $G_{\mathrm{R}}$ that is closest to the right of R1 and sets the index of the last sample in that block as the interpolation initialization sample where $k=0$. The algorithm then increments index $k$ from right to left until it identifies the last sample of the aligned signal, which is the index that satisfies the optimization:

$$
\begin{array}{ll}
\text { Minimize } & x_{k}-\left\|G_{\mathrm{R}}-\overleftrightarrow{R 1}\right\|^{\perp} \\
\text { subject to } & x_{k}=x_{k-1}+v_{k} \times \Delta \tau_{k}, k=\{1,2, \cdots\}
\end{array}
$$

and

$$
x_{0}=0
$$

Next, the algorithm interpolates the distance of the aligned traversal by setting the distance of the first sample to zero and ending the interpolation with the last sample.

\subsubsection{Midway Midpoint}

The pseudo-code for the algorithm is:

$$
\begin{aligned}
& \overleftrightarrow{C 0} \leftarrow \overleftrightarrow{R 0}+\frac{\overleftrightarrow{R 1}-\overleftrightarrow{R 0}}{2} \\
& \text { For } \forall j \in \mathbb{T} \\
& \quad \mathrm{G}_{\mathrm{M} j} \leftarrow \min _{i=\{0,1, \ldots\}}\left\|G_{i j}-\overleftrightarrow{C 0}\right\|^{\perp} \\
& \quad x_{K}^{\mathrm{M} j} \leftarrow x_{k}=x_{k-1}+v_{k} \times \Delta \tau_{k},\left\{x_{0}^{\mathrm{M} j}=0, k=\{0,1, \ldots\} \stackrel{\text { yields }}{\longrightarrow} x_{k}=\frac{x_{N-1}}{2}\right\}
\end{aligned}
$$


$\operatorname{Align}\left(x_{K}^{\mathrm{M} j}\right)$ :

$$
\begin{aligned}
& L_{j} \leftarrow x_{k}=x_{k-1}+v_{k} \times \Delta \tau_{k},\left\{x_{K}^{\mathrm{M} j}=0, k=\{0,-1, \ldots\}\right\} \\
& R_{j} \leftarrow x_{k}=x_{k-1}+v_{k} \times \Delta \tau_{k},\left\{x_{K}^{\mathrm{M} j}=0, k=\{0,+1, \ldots\}\right\} \\
& L_{\min } \leftarrow \min _{\forall j \in \mathbb{T}} L_{j}, R_{\min } \leftarrow \min _{\forall j \in \mathbb{T}} R_{j} \\
& x_{L j} \leftarrow \min _{\forall k}\left|x_{k j}-L_{\min }\right|, x_{R j} \leftarrow \min _{\forall k}\left|x_{k j}-R_{\min }\right|
\end{aligned}
$$

This method starts by spatially joining a centerline $\mathrm{C} 0$ that is at a distance that is midway between $\mathrm{R} 0$ and $\mathrm{R} 1$ and is perpendicular to the traversal path. The algorithm then locates the GPS block $\mathrm{G}_{\mathrm{M} j}$ that is closest to the centerline, on either side. The algorithm then sets the distance of the first sample in that GPS block to zero and interpolates to find the midpoint distance $x_{K}^{\mathrm{M} j}$ of that block. The Align procedure then sets the block's midpoint distance as the interpolation initialization sample where $k=0$ and interpolates distances to the left and right. The indices of the samples to the left and right of the interpolation initialization sample are negative and positive integers, respectively. Next, the procedure determines the minimum of the distances $L_{j}$ to the left of the center point as $L_{\min }$ and the minimum of the distances $R_{j}$ to the right of the center point as $R_{\min }$. Subsequently, the samples of each traversal with distances closest to $L_{\min }$ and $R_{\min }$ become the first and last samples, respectively, of the aligned traversal. The notation for the first and last samples of traversal $j$ is $x_{L j}$ and $x_{R j}$, respectively.

\subsubsection{Centroid Asymmetric}

This method identifies the single GPS block of each traversal that is closest to the $\mathrm{C} 0$ line and then calculates a centroid from the geospatial positions of that set of coordinates. Subsequently, the method spatially joins a line $\mathrm{C} 1$ that bisects the centroid and is parallel to $\mathrm{C} 0$. The algorithm then finds the left GPS block $G_{\mathrm{L} j}$ that is closest to $\mathrm{C} 1$ and then interpolates the distance from the first sample $x_{0}$ of that GPS block to find sample $x_{K}^{C j}$ that satisfies the optimization:

Minimize $\quad x_{K}^{C j} \leftarrow\left[x_{k j}-\left\|G_{\mathrm{L} j}-\overleftrightarrow{C 1}\right\|^{\perp}\right]$

subject to

and

$$
x_{k j}=x_{k j-1}+v_{k j} \times \Delta \tau_{k j}, k=\{0,1, \ldots\}
$$

$$
x_{0 j}=0 .
$$

The algorithm then calls the Align procedure with $x_{K}^{C j}$ as the input. The asymmetric aspect of this algorithm is that it selects the GPS block that is immediately to the left of the centroid rather than the GPS block that is closest on either side.

\subsubsection{Centroid Symmetric}

This method follows the procedure of the Centroid Asymmetric method with the exception that it starts with the GPS block that is closest to the centroid geofence, on either side. 


\subsubsection{Reference Method}

The authors previously introduced a method to estimate the distances of pavement distress from a known reference position on the road (Bridgelall, Huang, et al. 2016). While suitable for targeted roadway surveys, it is not practical to rely on known ground truths for widespread application of the method. The known ground truth produced the isolated PIE for each traversal (Figure 5). Therefore, the reference method can remove the position errors from GPS estimates to evaluate residual errors. This is achievable by interpolating distances to the left and right of the negative peak of each PIE, and then applying the Align procedure to set the first and last samples, respectively, of the aligned traversals. The residual errors are from estimates of the speed and sample intervals.

\subsection{Performance Evaluation}

The distribution of PIE distances and the lengths of the aligned signals from the output of each alignment algorithm provided a means for evaluating their relative performance. The distribution of the PIE distances from the beginning of the aligned traversals contained a large offset. Removing this offset improved the resolution of the histogram for statistical testing. The performance evaluation removed the offset by creating histograms of the PIE distances relative to the PIE distance of a common reference traversal. The traversal with the most samples served as the reference because having more samples for approximately equal distances achieves the highest resolution in distance estimates. The statistics compared were the mean, standard deviation (STD), and skewness, for the distribution of PIE distances and the lengths of the aligned traversals. Skewness is a measure of the amount of asymmetry of the distribution about its mean value. A standard normal distribution has a skewness of zero.

\subsection{Normality Tests}

The evaluation included normality tests of the PIE distributions resulting from each method of alignment. The normality tests included the Kolmogorov-Smirnov, Anderson-Darling, and Cramervon Mises tests (Yap and Sim 2011). These tests use an empirical distribution function (EDF) defined for a set of $n$ independent observations $\boldsymbol{X}_{1} \ldots \boldsymbol{X}_{\mathrm{n}}$ that have a common distribution function $\mathrm{F}(x)$. Under the null hypothesis, $\mathrm{F}(x)$ is the normal distribution. The tests reject the null hypothesis if the p-value of the test-statistic is less than 0.05 .

The empirical distribution function $(\mathrm{EDF}), \mathrm{F}_{\mathrm{n}}(\mathrm{x})$, takes a step of height $1 / \mathrm{n}$ at each observation such that

$$
F_{n}(x)= \begin{cases}0, & x<X_{(1)} \\ \frac{i}{n}, & X_{i} \leq x \leq X_{(i+1)}, i=1, \ldots, n-1 \\ 1, & X_{(n)} \leq x\end{cases}
$$

At any value $x, \mathrm{~F}_{\mathrm{n}}(x)$ is the proportion of observations less than or equal to $x$, while $\mathrm{F}(x)$ is the probability of an observation less than or equal to $x$. EDF statistics measure the discrepancy between $\mathrm{F}_{\mathrm{n}}(x)$ and $\mathrm{F}(x)$. The EDF tests use the probability integral transformation $U=\mathrm{F}(X)$ such that if $\mathrm{F}(\boldsymbol{X})$ is the distribution function of $\boldsymbol{X}$, then the random variable $U$ is uniformly distributed between 0 and 1 , where $U(i)=\mathrm{F}[X(i)]$ given $n$ observations $X(1), \ldots, X(\mathrm{n})$ are inputs for the EDF test statistics.

\subsubsection{Kolmogorov-Smirnov (KS) Test}

The Kolmogorov-Smirnov statistics (D) is 


$$
D=\sup _{x}\left|F_{n}(x)-F(x)\right|
$$

The Kolmogorov-Smirnov statistics belong to the supremum class of EDF statistics, which is based on the largest vertical difference between $\mathrm{F}(\mathrm{x})$ and $\mathrm{F}_{\mathrm{n}}(\mathrm{x})$. The Kolmogorov-Smirnov statistic is the maximum of D+ and D-, where D+ is the largest vertical distance between the EDF and the distribution function when the EDF is greater than the distribution function, and D- is the largest vertical distance when the EDF is less than the distribution function. That is,

$$
\begin{gathered}
D^{+}=\max _{i}\left(\frac{i}{n}-U_{(i)}\right) \\
D^{-}=\max _{i}\left(U_{(i)}-\frac{i-1}{n}\right) \\
D=\max \left(D^{+}, D^{-}\right) .
\end{gathered}
$$

\subsubsection{Anderson-Darling (AD) Test}

The Anderson-Darling statistic and the Cramer-von Mises statistic belong to the quadratic class of EDF statistics, which is based on the squared difference $\left(F_{n}(x)-F(x)\right)^{2}$. Quadratic statistics have the following general form:

$$
Q=n \int_{-\infty}^{+\infty}\left(F_{n}(x)-F(x)\right)^{2} \varphi(x) d F(x)
$$

The function $\varphi(x)$ weights the squared difference $\left[F_{n}(x)-F(x)\right]^{2}$. The Anderson-Darling statistics $\left(\mathrm{A}^{2}\right)$ is

$$
A^{2}=n \int_{-\infty}^{+\infty}\left[F_{n}(x)-F(x)\right]^{2}[F(x)(1-F(x))]^{-1} d F(x)
$$

Here the weight function is $\varphi(x)=[\mathrm{F}(x)(1-\mathrm{F}(x))]^{-1}$. The discrete form for computing the AndersonDarling statistic is

$$
A^{2}=-n-\frac{1}{n} \sum_{i=1}^{n}\left[(2 i-1) \log U_{(i)}+(2 n+1-2 i) \log \left(\left\{1-U_{(i)}\right\}\right]\right.
$$

\subsubsection{Cramer-von Mises (CVM) Test}

The Cramer-von Mises statistic $\left(\mathrm{W}^{2}\right)$ is

$$
W^{2}=n \int_{-\infty}^{+\infty}\left[F_{n}(x)-F(x)\right]^{2} d F(x) .
$$

Here the weight function is $\varphi(\mathrm{x})=1$. The discrete form for computing the Cramer-von Mises statistic is 


$$
W^{2}=\sum_{i=1}^{n}\left(U_{(i)}-\frac{2 i-1}{2 n}\right)^{2}+\frac{1}{12 n}
$$

\section{Results}

Figure 6 shows the histogram of the PIE distances for the reference method and the four methods of signal alignment. Table 2 summarizes the statistics of the evaluation.
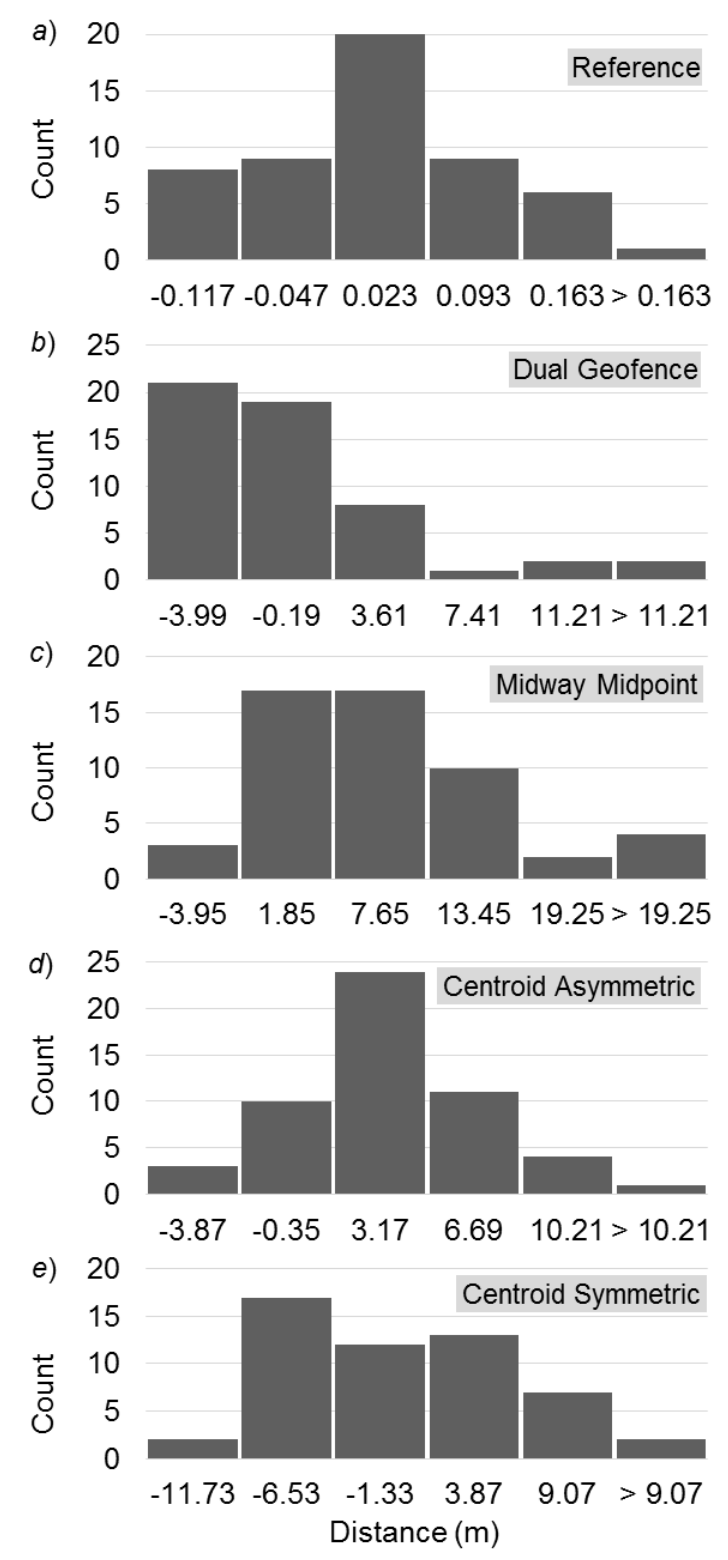

Figure 6. Distance distribution of the PIE.

The performance ranking is relative to the statistics that are closest to that of the reference method. Among the four methods, the Centroid Asymmetric provided the lowest standard deviation of the 
PIE and the least skewness. Except for the reference method, the Centroid Asymmetric method is the only one where none of the normality tests could reject the hypothesis that the distribution of the PIEs followed a normal distribution. The Centroid Asymmetric method also produced aligned signals with the lowest standard deviation of their lengths.

Table 2. Summary of Statistical Evaluation

\begin{tabular}{lccrrrrr}
\hline \multirow{2}{*}{ Method } & PIE & \multirow{2}{*}{ Skew } & \multicolumn{3}{c}{ P-Value } & Length \\
\cline { 5 - 6 } & STD & & KS & CVM & AD & STD \\
\hline Reference & 0.09 & 0.159 & $>0.150$ & $>0.250$ & $>0.250$ & 0.09 \\
Centroid Asymmetric & 4.04 & 0.335 & $>0.150$ & 0.128 & 0.149 & 0.13 \\
Dual Geofence & 4.84 & 1.546 & $<0.010$ & $<0.005$ & $<0.005$ & 5.59 \\
Centroid Symmetric & 6.32 & 0.522 & 0.101 & 0.099 & 0.080 & 0.24 \\
Midway Midpoint & 7.24 & 0.690 & 0.125 & 0.072 & 0.045 & 0.20 \\
\hline
\end{tabular}

Based on the standard deviation of the PIE, the Centroid Asymmetric method performed approximately 17\%, 36\%, and 44\% better than the Dual Geofence, Centroid Symmetric, and Midway Midpoint methods, respectively. As noted earlier, the GPS position spread of PIEs along the traversal path was 30.4 meters, which is approximately equivalent to a standard deviation of 5.1 meters. Hence, the Centroid Asymmetric method improved the standard deviation over GPS estimates by approximately one meter or $20 \%$. The reference method determined that interpolation errors from speed and time estimates accounted for less than 10 centimeters of the standard deviation, which is less than $2 \%$ of the GPS standard deviation.

\section{Discussion}

Based on knowing the ground truth, it was possible to measure the GPS error spread in two dimensions. Even though the sky was clear and there were no obstructions such as trees to interfere with the line-of-sight conditions, the GPS error spread from this experiment was much larger than commonly expected. The best algorithm provided a significant improvement over the GPS spread without relying on robust GPS measurements along the traversal path. The distance distribution of the PIEs from the best-performing algorithm had relatively low skew and exhibited no significant departure from normality. Based on general statistical principles, normality suggests that the algorithm will improve the alignment continuously as additional data become available from more traversals. Hence, the approach is well suited for connected vehicle environments.

There were two surprising outcomes. First, although the Dual Geofence and Centroid Symmetric methods attempted to select interpolation initialization samples from GPS blocks that were closest to a geofence they were not the top performers. Second, although the two centroidbased algorithms are nearly identical, the symmetric method performed worse than the asymmetric method that selected interpolation initialization samples from GPS blocks that were closest to the geofence. A possible explanation for this phenomenon is that biasing the selection of GPS blocks to one side of a geofence more closely matches the distribution bias of GPS errors from a vehicle moving in one direction along a path. It was less surprising that the Midway Midpoint algorithm performed worst because it randomly selected the block center sample for interpolation initialization.

There are three practical considerations when using the best-performing algorithm. Firstly, since it uses a single geofence that bisects a centroid of GPS position updates, the algorithm can simply begin with a single geofence instead of first identifying two reference blocks to extract 
traversal data. That is, the algorithm may select GPS blocks that are close to a geospatial point of interest, such as a milepost, compute the centroid position, and then interpolate distances outwards on either side of the bisecting perpendicular, and stop when the distances are approximately equal. This method is robust to GPS errors and missing GPS updates because the centroid is an average of the geospatial position estimates from many traversals. Furthermore, the method can detect and remove GPS outliers before computing the centroid. The second consideration is that the algorithm should assign the zero-distance position of each aligned traversal to the same geospatial position on the traversal path. This is achievable by finding the centroid from all GPS blocks that are associated with the first inertial sample of the aligned traversals, and then creating a bisecting perpendicular to the traversal path. The third consideration is that since the distance interpolation provides a very

high position resolution, methods of extracting features from equal length distance windows will be more tolerant to residual alignment errors. Feature extraction methods can range from simple distance averaging to more complex space-time methods such as the Gabor or wavelet transforms. However, a more detailed discussion of feature extraction algorithms will dilute the focus of this paper.

\section{Conclusion}

Connected vehicle environments provide an opportunity to detect roadway and railway anomalies with low error rates by using signal combination techniques. However, the low resolution and large variations in GPS position estimates create signal misalignment and gaps that can reduce the quality of the composite signal, and thus increase detection errors. To resolve these issues, the authors invented and evaluated four methods of distance tagging that reduced the reliance on GPS position estimates along traversals. Each method used distance interpolation with velocity and sample period updates to increase the position resolution substantially, and consequently improve signal alignment. Distance interpolation also accommodates the non-uniform sampling of the inertial signals. Furthermore, the very high resolution from millisecond updates of the inertial sensors reduces the errors from asynchronous spatial sampling.

The best method provided a $20 \%$ improvement in signal alignment over GPS measurements. Based on the standard deviation of a large inertial event, the best method performed approximately $17 \%$ and $44 \%$ better than the next best and worst methods, respectively. There was no significant departure from normality for the distance distribution of the reference inertial event, and the distribution skewness was relatively low. These statistical properties suggest that the signal quality will improve continuously as more data becomes available from traversals in a connected vehicle environment. Based on knowing the position of a ground truth to remove GPS errors, interpolation errors from speed and time estimates accounted for less than $2 \%$ of the standard deviation of distance estimates.

The method is easy to use in practice, has low computational complexity, and is robust to GPS errors and missing GPS updates. That is, the method requires finding a single centroid from a cluster of GPS position updates near a position of interest, and then interpolating distances outwards from that point by using speed and sample period updates from the inertial sensors. Lateral GPS errors that deviate from the travel path do not worsen errors because the method measures distances from a centroid bisecting line that is perpendicular to the traversal path. 


\section{Acknowledgement}

Funds from the North Dakota State University and the Mountain-Plains Consortium, a University Transportation Center funded by the U.S. Department of Transportation supported this work. The contents of this paper reflect the views of the authors, who are responsible for the facts and accuracy of the information presented.

\section{References}

Aldrich, Mark. 2018. Back on Track: American Railroad Accidents and Safety, 1965-2015. Baltimore: John Hopkins University Press.

Bajaj, Rashmi, Samantha Lalinda Ranaweera, and Dharma P. Agrawal. 2002. "GPS: locationtracking technology." Computer 35 (4): 92-94. doi:10.1109/MC.2002.993780.

Bernal, Esteban, Maksym Spiryagin, and Colin Cole. 2018. "Onboard condition monitoring sensors, systems and techniques for freight railway vehicles: a review." IEEE Sensors Journal 19 (1): 4-24. doi:10.1109/JSEN.2018.2875160.

Bridgelall, Raj. 2014. "Connected Vehicle Approach for Pavement Roughness Evaluation." Journal of Infrastructure Systems (American Society of Civil Engineers) 20 (1): 04013001 (1-6). doi:10.1061/(ASCE)IS.1943-555X.0000167.

Bridgelall, Raj. 2014. "Inertial Sensor Sample Rate Selection for Ride Quality Measures." Journal of Infrastructure Systems (American Society of Civil Engineering) 21 (2): 04014039 (1-5). doi:10.1061/(ASCE)IS.1943-555X.0000225.

Bridgelall, Raj, Md Tahmidur Rahman, Denver Tolliver, and Jerome F. Daleiden. 2019.

"Wavelength sensitivity of roughness measurements using connected vehicles." International Journal of Pavement Engineering (Taylor and Francis) 20: 566-572. doi:10.1080/10298436.2017.1316645.

Bridgelall, Raj, Ying Huang, Zhiming Zhang, and Fudan Deng. 2016. "Precision enhancement of pavement roughness localization with connected vehicles." Measurement Science and Technology 27 (2): 025012. doi:10.1088/0957-0233/27/2/025012.

Chen, Chi-Tsong. 2004. Signals and Systems. 3rd. New York: Oxford University Press.

Chen, Qijin, Xiaoji Niu, Lili Zuo, Tisheng Zhang, Fuqin Xiao, Yi Liu, and Jingnan Liu. 2018. "A railway track geometry measuring trolley system based on aided INS." Sensors 18 (2): 538. doi:10.3390/s 18020538.

Chia, Leonard, Bhavana Bhardwaj, Pan Lu, and Raj Bridgelall. 2018. "Railroad Track Condition Monitoring Using Inertial Sensors and Digital Signal Processing: A Review." IEEE Sensors Journal 19 (1): 25-33. doi:10.1109/JSEN.2018.2875600.

Dennis, Eric Paul, Qiang Hong, Richard Wallace, William Tansil, and Matt Smith. 2014. "Pavement condition monitoring with crowdsourced connected vehicle data." Transportation Research Record 2460 (1): 31-38. doi:10.3141/2460-04.

Dertimanis, Vasileios K., Manuel Zimmermann, Francesco Corman, and E. N. Chatzi. 2019. "OnBoard Monitoring of Rail Roughness via Axle Box Accelerations of Revenue Trains with 
Uncertain Dynamics." Edited by Barthorpe R. Model Validation and Uncertainty Quantification. Cham: Springer. doi:10.1007/978-3-030-12075-7_18.

El-Wakeel, Amr S., Jin Li, Aboelmagd Noureldin, Hossam S. Hassanein, and Nizar Zorba. 2018. "Towards a practical crowdsensing system for road surface conditions monitoring." IEEE Internet of Things Journal 5 (6): 4672-4685. doi:10.1109/JIOT.2018.2807408.

GAO. 2019. Positive Train Control: As Implementation Progresses, Focus Turns to the Complexities of Achieving System Interoperability. Congressional Testimony, Washington, D.C.: U.S. Government Accountability Office. https://www.gao.gov/products/GAO-19-693t.

Gikas, Vassilis, and Harris Perakis. 2016. "Rigorous performance evaluation of smartphone GNSS/IMU sensors for ITS applications." Sensors 16 (8): 1240. doi:10.3390/s16081240.

Groves, Paul D., Lei Wang, and M. Ziebart. 2012. "Shadow Matching: Improved GNSS Accuracy in Urban Canyons." GPS World 23 (2): 14-18.

Hughes, William J. 2016. Global Positioning System (GPS) Standard Positioning Service (SPS) Performance Analysis Report. Performance Analysis Report 94, T and E Team, Federal Aviation Administration (FAA), Wide Area Augmentation System (WAAS), Washington, D.C.: Technical Center.

Hunter, Timothy, Ryan Herring, Pieter Abbeel, and Alexandre Bayen. 2009. "Path and travel time inference from GPS probe vehicle data." NIPS Analyzing Networks and Learning with Graphs. Vancouver, Canada: Neural Information Processing Systems (NIPS) Foundation. 18.

Jo, Youngtae, and Seungki Ryu. 2015. "Pothole detection system using a black-box camera." Sensors 15 (11): 29316-29331. doi:10.3390/s151129316.

Ma, Junhu, Jinwen Xie, and Lu Gan. 2018. "Compressive detection of unknown-parameters signals without signal reconstruction." Signal Processing (Elsevier) 142: 114-118. doi:10.1016/j.sigpro.2017.07.010.

Medeiros, Leandro, Pedro Henrique Oliveira Silva, Lucas de Castro Valente, and Erivelton Geraldo Nepomuceno. 2018. "A Prototype for Monitoring Railway Vehicle Dynamics Using Inertial Measurement Units." 2018 13th IEEE International Conference on Industry Applications (INDUSCON). IEEE. 149-154. doi:10.1109/INDUSCON.2018.8627330.

Moschas, Fanis, Panos A. Psimoulis, and Stathis C. Stiros. 2013. "GPS/RTS data fusion to overcome signal deficiencies in certain bridge dynamic monitoring projects." Smart structures and systems 12 (3-4): 251-269. doi:10.12989/sss.2013.12.3_4.251.

OBrien, Eugene J., Paraic Quirke, Cathal Bowe, and Daniel Cantero. 2018. "Determination of railway track longitudinal profile using measured inertial response of an in-service railway vehicle." Structural Health Monitoring 17 (6): 1425-1440. doi:10.1177/1475921717744479.

Paixão, André, Eduardo Fortunato, and Rui Calçada. 2019. "Smartphone's Sensing Capabilities for On-Board Railway Track Monitoring: Structural Performance and Geometrical Degradation Assessment." Advances in Civil Engineering 2019: 13. doi:10.1155/2019/1729153. 
Pierce, Linda M., and Nicholas D. Weitzel. 2019. NCHRP Synthesis 531: Automated Pavement Condition Surveys. NCHRP Synthesis, Washington, D.C.: National Cooperative Highway Research Program (NCHRP).

Stiros, Stathis C., and Panos A. Psimoulis. 2012. "Response of a historical short-span railway bridge to passing trains: 3-D deflections and dominant frequencies derived from Robotic Total Station (RTS) measurements." Engineering Structures (Elsevier) 45 (December 2012): 362371. doi:10.1016/j.engstruct.2012.06.029.

USDOT. 2015. Beyond Traffic 2045: Trends and Choices. Washington, D.C.: United States Department of Transportation.

Yap, Bee Wah, and Chiaw Hock Sim. 2011. "Comparisons of various types of normality tests." Journal of Statistical Computation and Simulation 81 (12): 2141-2155. doi:10.1080/00949655.2010.520163. 